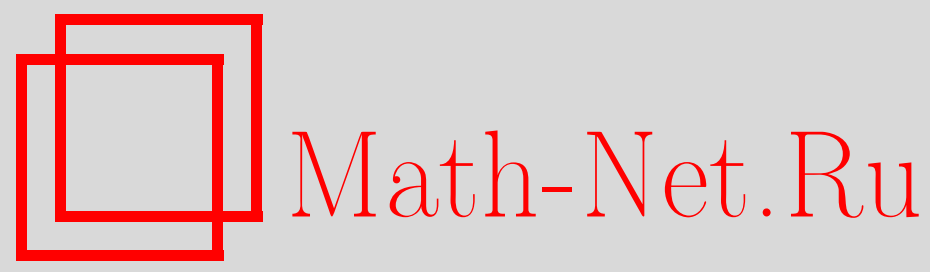

И. П. Павлоцкий, М. Стрианезе, О локальной устойчивости экстремумов интегральных кривых ОДУ второго порядка, Матем. заметки, 2002, том 71, выпуск 5, 742-750

DOI: https://doi.org/10.4213/mzm382

Использование Общероссийского математического портала Math-Net.Ru подразумевает, что вы прочитали и согласны с пользовательским соглашением http://www.mathnet.ru/rus/agreement

Параметры загрузки:

IP : 54.80 .97 .219

26 апреля 2023 г., 14:50:57

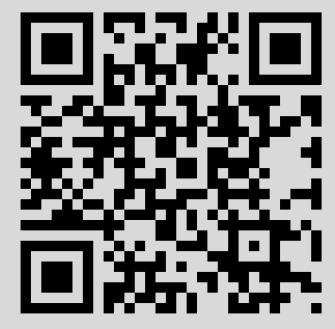




\title{
О ЛОКАЛЬНОЙ УСТОЙЧИВОСТИ ЭКСТРЕМУМОВ ИНТЕГРАЛЬНЫХ КРИВЫХ ОДУ ВТОРОГО ПОРЯДКА
}

\author{
И.П. Павлоцкий, М. Стрианезе
}

В [1]-[3] определено продолжение решения уравнения $a(x, \dot{x}) \ddot{x}=1, x \in \mathbb{R}, a(x, \dot{x}) \in$ $C^{1}$, на сингулярное множество $S=\left\{(x, y) \in \mathbb{R}^{2}: a(x, y)=0\right\}, y=\dot{x}$, через первый интеграл. В этом случае все стационарные точки и все локальные экстремумы интегральной кривой $x(y)$ такие, что в экстремальной точке $x(y)$ имеет производную, находятся на множестве $S \cup Y$, где $Y$ - множество точек прямой $y=0$. В работе изучается локальная устойчивость типов локальных экстремумов при переходе к уравнению $[a(x, y)+\varepsilon b(x, y)] \dot{y}=1, b(x, y) \in C^{1}$, при достаточно малом $|\varepsilon|$. Обозначая $S^{*}=\left\{(x, y) \in \mathbb{R}^{2}: a(x, y)+\varepsilon b(x, y)=0\right\}$, мы условно говорим об устойчивости типов локальных экстремумов относительно замены $S$ на $S^{*}$. Найдены некоторые достаточные условия устойчивости и неустойчивости.

Библиография: 12 названий.

1. Введение. Работа посвящена частному вопросу качественной теории ОДУ. В ней изучается устойчивость относительно расположения локальных экстремумов на интегральных кривых $x(y)$ и $x^{*}(y)$, возникающих соответственно из систем

$$
a(x, y) \frac{d y}{d t}=1, \quad \frac{d x}{d t}=y, \quad x \in \mathbb{R}, \quad y \in \mathbb{R}, \quad a(x, y) \in C^{1}, \quad t \in T \subseteq \mathbb{R},
$$

и

$$
[a(x, y)+\varepsilon b(x, y)] \frac{d y}{d t}=1, \quad x \in \mathbb{R}, \quad y \in \mathbb{R}, \quad a(x, y) \in C^{1}, \quad t \in T^{*} \subseteq \mathbb{R}, \quad \varepsilon \in \mathbb{R}
$$

при достаточно малом $|\varepsilon|$. Случай $a(x, y) d y / d t=0$ не рассматривается. Заметим, что обычно изучается функция $y(x)$, так как, зная $y=f(x)$, т.е. $d x / d t=f(x)$, сразу получаем квадратуру $t=\int d x / f(x)$. Однако исследование свойств $x(y)$ не только дополняет общую картину, но иногда оказывается более важным в приложениях (см., например, $[4]-[7])$.

Будем называть $\left(x_{0}, y_{0}\right)$ стационарной точкой функции $x(y)$, если $d x / d y=0$ при $y=y_{0}$ и $x=x_{0}$. Множество $S t$ всех стационарных точек назовем стационарным множсеством. Аналогично для $x^{*}(y)$. Назовем $\left(x_{0}, y_{0}\right)$ әкстремальной точкой функции $x(y)$, если $x(y)$ имеет при $y=y_{0}$ локальный экстремум и $x_{0}=x\left(y_{0}\right)$. Аналогично для $x^{*}(y)$. 
ОПРЕДЕЛЕНИЕ 1. $S=\left\{(x, y) \in \mathbb{R}^{2}: a(x, y)=0\right\}$ есть сингулярное множество системы (1), а $S^{*}=\left\{(x, y) \in \mathbb{R}^{2}: a(x, y)+\varepsilon b(x, y)=0\right\}-$ сингулярное мнохество системы (2). Точки множеств $S$ и $S^{*}$ назовем сингулярными.

Для краткости обозначим через $Y$ множество всех точек прямой $y=0$ в декартовой системе координат на плоскости. Как видно из (1) и (2),

$$
\frac{d x}{d y}=y a(x, y) \in C^{1}, \quad \frac{d x^{*}}{d y}=y[a(x, y)+\varepsilon b(x, y)] \in C^{1}
$$

и, следовательно, если $x(y)$ можно продолжить на $S$ так, чтобы для любой точки $(x(y)$, $y) \in S$ вьполнялось равенство $a(x(y), y)=0$, а $x^{*}(y)$ можно продолжить на $S^{*}$ так, чтобы для любой точки $\left(x^{*}(y), y\right) \in S$ имело место $a\left(x^{*}(y), y\right)+\varepsilon b\left(x^{*}(y), y\right)=0$, то $S t=S \cup Y$, а $S t^{*}=S^{*} \cup Y$, где $S t^{*}$ - стационарное множество, отвечающее уравнению (2). Такое продолжение интегральной кривой на $S \cup Y$ (на $S^{*} \cup Y$ ) через продолжение на $S \cup Y\left(\right.$ на $S^{*} \cup Y$ ) в классе $C^{1}$ первого интеграла было определено и изучалось в [1]-[3]. Очевидно, первые интегралы не определены на сингулярных множествах системами (1) и (2).

Предположим, что первый интеграл $I(x, y)$, соответствующий $(1)$, и первый интеграл $G(x, y)$, соответствующий системе (2), можно продолжить на $S$ и $S^{*}$ в классе $C^{1}$. Продолжение будем обозначать также: $I$ и $G$. Продолжение траектории определим только для системы (1), так как определение для случая (2) получается дословньм повторением.

ОПРЕДЕЛЕниЕ 2. Пусть $[\varphi(t), \dot{\varphi}(t)] \in M \subseteq \mathbb{R}^{2}, t \in T,-$ траектория, определяемая системой $(1) ;[x(t), y(t)]$ называется продолжением этой траектории на $M \cup S$ через $I(x, y)$, если $\forall t \in T: x(t)=\varphi(t), y(t)=\dot{\varphi}(t)$ и $I(x(t), y(t)) \equiv C$ на $M \cup S$.

Будем предполагать mes $S=0$ относительно лебеговой меры в $\mathbb{R}^{2}$.

В рассматриваемых условиях локальный первьй интеграл $I$ всегда существует в $O \backslash S$, где $O$ - некоторая окрестность точки $\left(x_{0}, y_{0}\right) \in S[8]$, но, разумеется, отсюда не следует его продолжаемость на $S$ или его часть в классе $C^{1}$. Та же ситуация имеет место для уравнения (2). Заметим, что по локальной теореме Дини о неявной функции если $I_{x}\left(x_{0}, y_{0}\right) \neq 0$, то интеграл $\widetilde{I}=I(x, y)-I\left(x_{0}, y_{0}\right)$ определяет в некоторой окрестности точки $\left(x_{0}, y_{0}\right)$ единственную функцию $x=f(y)$. В общем сучае ситуация может быть намного сложнее [1], [2].

Для некоторого сокращения дальнейших формулировок будем считать, что во всех дальнейших рассуждениях выполняются следующие

УСловия. $a(x, y) \in C^{1}, b(x, y) \in C^{1}, \varepsilon \in \mathbb{R}, I(x, y) \in C^{1}, G(x, y) \in C^{1} ; I_{x}\left(x_{0}, y_{0}\right) \neq 0$, $\left(x_{0}, y_{0}\right) \in S ; G_{x}\left(x^{*}, y^{*}\right) \neq 0,\left(x^{*}, y^{*}\right) \in S^{*}$.

Вообще говоря, тип локального экстремума функции $x(y)$ (или $\left.x^{*}(y)\right)$ не определяет единственным образом продолжение траектории через сингулярную точку. Как показано в [1], [2], сингулярная точка может быть концом траектории, началом с двумя возможными продолжениями, а также точкой, откуда траектория делает скачок в другой локальный экстремум (тогда $x(t)$ (или $x^{*}(t)$ ) определена только в классе обобщенных функций). Таким образом, изучение интегральной кривой помогает понять свойства 
трактории, но требуется некоторое дополнительное рассуждение. Важно то, что устойчивость интегральной кривой на $S \cup Y\left(\right.$ на $S^{*} \cup Y$ ) ведет к устойчивому продолжению траектории, и наоборот.

Мы говорим о двух типах устойчивости при переходе от уравнения (1) к уравнению (2). Устойчивость назьвается топологической, если $S$ и $S^{*}$ имеют одинаковую топологию при достаточно малом $|\varepsilon|$. В настоящей работе рассматривается только устойчивость локальных экстремумов интегральньх кривых при переходе от (1) к (2) для достаточно малого $|\varepsilon|$ (ЛЭ-устойчивость). Общее определение ЛЭ-устойчивости дается в работах [1]-[3]. В следующем пункте мы ограничимся изучением локальной ЛЭ-устойчивости и дадим ее определение, являющееся частным случаем общего.

В работах [9], [10] (см. также [11]) изучались оба типа устойчивости в частном сучае, когда $a(x, y)$ - полином не вьше второго порядка; в [9] для $b=1$, а в [10] для $b=x$ и $b=y$. Оказалось, что неустойчивость может появиться только при вырожденных кривых второго порядка (эллипс, гипербола и парабола квазиустойчивы). Тип неустойчивости зависит также от расположения прямых относительно декартовьх координатных осей и, разумеется, от характера самих прямых возмущений.

2. Некоторые теоремы о локальной ЛЭ-устойчивости. Любой тип неустойчивости радикально меняет качественную картину поведения интегральных кривых и траекторий, достигающих сингулярного множества, если уравнение подвергнуто малому возмущению.

ОПРЕДЕЛЕНИЕ 3 . Точка $\left(x_{0}, y_{0}\right) \in S \cup Y$ является точкой локальной ЛЭ-устойчивости, если для любого достаточно малого $\varepsilon>0$ сушествует окрестность $O\left(x_{0}, y_{0}\right)$ этой точки такая, что в этой окрестности типы локальных экстремумов функций $x(y)$ и $x^{*}(y)$ (т.е. интегральных кривых уравнений (1) и (2)) одинаковы на $(S \cup Y) \cap O$ и на $\left(S^{*} \cup Y\right) \cap O$.

Как следует из введения (см. также формулы (3)), локальные экстремумы кривой $x(y)$, где она дифференцируема, могут существовать только на $S \cup Y$, а локальные экстремумы кривой $x^{*}(y)$, отвечающей уравнению $(2)$, располагаются только на $S^{*} \cup Y$. Нам понадобится несколько производных функций $x(y)$ и $x^{*}(y)$.

Из (3) следует, что на $S \cup Y$

$$
\frac{d x}{d y}=0, \quad \frac{d^{2} x}{d y^{2}}=a(x, y)+y a_{y}(x, y)
$$

Действительно,

$$
\left.\frac{d}{d y} y a(x(y), y)\right|_{S \cup Y}=\left[a+y\left(a_{x} \frac{d x}{d y}+a_{y}\right)\right]_{S \cup Y} .
$$

Если, кроме того, $a_{y y}$ ограничена на $S \cup Y$, из аналогичных вычислений следует, что на $S \cup Y$

$$
\frac{d^{3} x}{d y^{3}}=2 a_{y}+y\left(y a_{x} a_{y}+a_{y y}\right)
$$

откуда вытекает, что на $S \cup Y$

$$
\frac{d^{3} x}{d y^{3}}=2 a_{y}
$$


Соответствующие производные функции $x^{*}(y)$ будут написаны в тексте доказательств. Заметим, что если $a(x, y) \in C^{\infty}$, то с помощью одних только производных $a(x, y)$ по $y$ в точках множества $S \cup Y$ всегда можно установить, имеет ли $x(y)$ в этих точках локальный экстремум и тип экстремума [12].

Установим достаточные условия локальной ЛЭ-устойчивости.

ТЕОрема 1. Пусть в полуплоскости $y>0$ или $y<0$ точка $\left(x_{0}, y_{0}\right) \in S u$ $a_{y}\left(x_{0}, y_{0}\right) \neq 0$. Тогда $\left(x_{0}, y_{0}\right)$ - точка локальной ЛЭ-устойчивости.

ДокАзАТЕЛьство. На $S \cup Y d x / d y=0$, а на $S^{*} \cup Y d x^{*} / d y=0$. По условиям теоремы и по формуле $(3 \mathrm{a})$ в точке $\left(x_{0}, y_{0}\right) \in S$

$$
\frac{d^{2} x}{d y^{2}}=y_{0} a_{y}\left(x_{0}, y_{0}\right) \neq 0 \text {. }
$$

Вычисляя вторую производную интегральной кривой $x^{*}(y)$ на $S^{*}$, получаем

$$
\frac{d^{2} x^{*}}{d y^{2}}=y\left(a_{y}(x, y)+\varepsilon b_{y}(x, y)\right), \quad(x, y) \in S^{*} .
$$

Итак, $\left(x_{0}, y_{0}\right)$ - экстремальная точка функции $x(y)$. Из условия $a(x, y) \in C^{1}$ следует, что существует ограниченная окрестность $O\left(x_{0}, y_{0}\right)$ точки $\left(x_{0}, y_{0}\right)$, не пересекающаяся c $Y$, где $a_{y}(x, y)$ сохраняет знак. Покажем, что для любого достаточно малого $|\varepsilon|$ сушествует окрестность $O^{*}\left(x_{0}, y_{0}\right) \subseteq O\left(x_{0}, y_{0}\right)$ такая, что знак $d^{2} x^{*} / d y^{2}$ в $O^{*} \cap S^{*}$ совпадает со знаком $d^{2} x / d y^{2}\left(x_{0}, y_{0}\right)$. Выберем $O^{*}$ так, чтобы в ней выполнялось $\left|a_{y}(x, y)\right| \geqslant$ $\left|a_{y}\left(x_{0}, y_{0}\right)\right| / 2$, и обозначим

$$
B=\sup _{\bar{O}^{*}}\left|b_{y}(x, y)\right|
$$

где $\bar{O}^{*}$ - замыкание $O^{*}$. В тривиальном случае $B=0$ производная $d^{2} x^{*} / d y^{2}$ сохраняет знак всюду в $O^{*} \cap S^{*}$ для любого $\varepsilon$. Если же $B \neq 0$, ее знак не меняется всюду в $O^{*}$ при условии $\left|a_{y}\left(x_{0}, y_{0}\right)\right| / 2>|\varepsilon| B$, т.е. для любого $\varepsilon$, удовлетворяющего условию $0<|\varepsilon|<\left|a_{y}\left(x_{0}, y_{0}\right)\right| /(2 B)$.

Однако может оказаться, что при этих условиях в $\bar{O}^{*}$ нет ни одной точки множества $S^{*}$. Покажем, что $\bar{O}^{*} \cap S^{*} \neq \varnothing$, если $|\varepsilon|$ достаточно мал. Рассмотрим прирашение $\Delta a=a(x, y)-a\left(x_{0}, y_{0}\right)=a(x, y)$. Из условия $a_{y}\left(x_{0}, y_{0}\right) \neq 0$ следует, что не существует такой окрестности точки $\left(x_{0}, y_{0}\right)$, где бы всюду имело место либо $\Delta a=0$, либо $\Delta a<0$, либо $\Delta a>0$. Действительно, условие $\Delta a<0$ (или $\Delta a>0$ ) означало бы, что $\left(x_{0}, y_{0}\right)$ - точка локального экстремума функции $a(x, y)$, что противоречит условию теоремы $a_{y}\left(x_{0}, y_{0}\right) \neq 0$. Очевидно, $\Delta a=0$ также невозможно. Иными словами, в любой окрестности точки $\left(x_{0}, y_{0}\right)$ (в частности, в $\left.O^{*}\left(x_{0}, y_{0}\right)\right) a(x, y)$ принимает значения обоих знаков. Обозначим

$$
\beta=\sup _{\bar{O}^{*}}|b(x, y)|, \quad \varepsilon_{0}=\min \left(\left|\inf _{\bar{O}^{*}} a(x, y)\right|, \sup _{\bar{O}^{*}} a(x, y)\right)
$$

и возмем значения $\varepsilon$ такие, что $\varepsilon_{0}>|\varepsilon| \beta$. В случае $\beta=0$ можно взять любое $\varepsilon_{0}>0$, т.е. нетривиален случай $\beta \neq 0$. Из непрерывности функций $a(x, y)$ и $b(x, y)$ вытекает, что в $\bar{O}^{*} \forall \varepsilon\left(0<|\varepsilon|<\varepsilon_{0} / \beta\right) \exists(x, y): \Delta a=a(x, y)=-\varepsilon b(x, y)$, т.е. $a(x, y)+\varepsilon b(x, y)=0 \Longrightarrow$ $(x, y) \in S^{*}$. Действительно, $a(x, y)$ принимает все значения из отрезка $\left[-\varepsilon_{0}, \varepsilon_{0}\right]$.

Таким образом, в $\bar{O}^{*}$ типы локальных экстремумов $x(y)$ на $S$ и $x^{*}(y)$ на $S^{*}$ совпадают, если $0<|\varepsilon|<\min \left[a_{y}\left(x_{0}, y_{0}\right) /(2 B), \varepsilon_{0} / \beta\right]$.

С помощью совершенно аналогичных рассуждений может быть доказана 
Теорема 2. Пусть в полуплоскости $у>0$ (или $y<0)$ точка $\left(x_{0}, y_{0}\right) \in S$, $a_{y}\left(x_{0}, y_{0}\right)=0 u b_{y}\left(x_{0}, y_{0}\right) \neq 0$. Тогда $\left(x_{0}, y_{0}\right)$ не является точкой локальной ЛЭ-устойчивости.

Теперь рассмотрим точку $\left(x_{0}, 0\right) \in Y$, не принадлежащую сингулярному множеству.

Teорема 3. Пусть точка $\left(x_{0}, 0\right) \notin S$ и $O\left(x_{0}, 0\right)$ - окрестность, где $a\left(x_{0}, 0\right) \neq 0$ сохраняет знак. Тогда для любой функиии $b(x, y) \in C^{1}$ не существует числа $\varepsilon_{0}$ такого, что из условия $0<|\varepsilon|<\varepsilon_{0}$ следует, что $S^{*} \cap O \neq \varnothing$.

ДокАЗАТЕЛЬСТво. Из непрерывности $a(x, y)$ следует существование окрестности $O\left(x_{0}, 0\right)$ точки $\left(x_{0}, 0\right)$, в которой $a(x, y)$ имеет тот же знак, что и $a\left(x_{0}, 0\right)$. Пусть для определенности $a(x, y)>0$ в $O$.

a) Если $b(x, y)=0$ в точке $(x, y) \in O$, то $a(x, y)+\varepsilon b(x, y)>0 \Longrightarrow(x, y) \notin S^{*}$.

b) Если $b(x, y)>0$ в $(x, y) \in O$, то для любого $\varepsilon>0 a(x, y)+\varepsilon b(x, y)>0 \Longrightarrow$ $(x, y) \notin S^{*}$.

c) Если $b(x, y)<0$ в $(x, y) \in O$, то для любого $\varepsilon<0 a(x, y)+\varepsilon b(x, y)>0 \Longrightarrow$ $(x, y) \notin S^{*}$.

Аналогично в случае $a(x, y)<0$ в $O$.

Теперь рассмотрим точку в пересечении $S \cap Y$.

Tеорема 4. Пусть точка $\left(x_{0}, 0\right) \in S \cap Y, a_{x}\left(x_{0}, 0\right) \neq 0, a_{y}\left(x_{0}, 0\right) \neq 0 u a_{y y}$ ограничена на $S^{*} \cup Y$. Тогда на оси $Y$ существует одномерная окрестность точки $\left(x_{0}, 0\right)$, где интегральные кривые $x(y)$ u $x^{*}(y)$ (отвечающие уравнениям (1) $u(2)$ ) при достаточно малом $|\varepsilon|$ либо одновременно возрастают, либо одновременно убывают, проходя через любую точку окрестности.

ДокАЗАТЕЛьСтво. Теорема доказывается в классе $a(x, y) \in C^{2}$.

По условиям теоремы и по формулам (3) и (3a) на $S \cap Y$

$$
\frac{d x}{d y}=\frac{d^{2} x}{d y^{2}}=0
$$

а на $S^{*} \cap Y$

$$
\frac{d x^{*}}{d y}=\frac{d^{2} x^{*}}{d y^{2}}=0
$$

По формулам (3) и (3b) на $S \cap Y$

$$
\frac{d^{3} x}{d y^{3}}\left(x_{0}, 0\right)=2 \frac{\partial a}{\partial y}\left(x_{0}, 0\right) \neq 0
$$

а на $S^{*} \cap Y$

$$
\frac{d^{3} x^{*}}{d y^{3}}(x, 0)=2 \frac{\partial(a+\varepsilon b)}{\partial y}(x, 0),
$$

поскольку по условию теоремы $a_{y y}$ ограничена на $S^{*} \cap Y$.

Из непрерывности $a_{y}(x, y)$ следует существование ограниченной окрестности $O\left(x_{0}, 0\right)$ точки $\left(x_{0}, 0\right)$, где $a_{y}(x, y)$ и как следствие $d^{3} x / d y^{3}$ сохраняет знак. Пусть $\bar{O}_{1}\left(x_{0}\right) \subseteq O$ - 
отрезок оси $Y$, содержащий внутри себя $x_{0}$ и определенньй условием $\left|a_{y}(x, 0)\right|>\mid a_{y}\left(x_{0}\right.$, $0) \mid / 2$. Обозначим

$$
B=\sup _{\bar{O}_{1}}\left|b_{y}(x, y)\right|
$$

Предположим, что интегральная кривая $x^{*}(y)$ проходит через $\bar{O}_{1}$ (ниже мы докажем это для любого достаточно малого $|\varepsilon|)$. В тривиальном случае $B=0 d^{3} x / d y^{3}$ и $d^{3} x^{*} / d y^{3}$ совпадают во всех точках $\bar{O}_{1}$. Если же $B \neq 0$, эти третьи производные имеют всюду в $\bar{O}_{1}$ одинаковые знаки, когда $\left|a_{y}\left(x_{0}, 0\right)\right| / 2>|\varepsilon| B$. Тогда функции $x(y)$ и $x^{*}(y)$ либо возрастают в любой точке $\bar{O}_{1}$, либо убывают в любой точке $\bar{O}_{1}$ в зависимости от знака $a_{y}\left(x_{0}, 0\right)$.

Покажем, что кривая $x^{*}(y)$ действительно попадает в $\bar{O}_{1}$, если $|\varepsilon|$ достаточно мал, т.е. $\bar{O}_{1} \cap S^{*} \neq \varnothing$. Обозначим

$$
\beta=\sup _{\bar{O}_{1}}|b(x, 0)|
$$

В тривиальном случае $\beta=0$, очевидно, $\bar{O}_{1} \cap S=\bar{O}_{1} \cap S^{*}$. Если $\beta \neq 0$, то из непрерывности функций $a(x, 0): \mathbb{R} \rightarrow \mathbb{R}$ и $b(x, 0): \mathbb{R} \rightarrow \mathbb{R}$ следует, что уравнение $a(x, 0)+\varepsilon b(x, 0)$ $=0$ имеет решения при условии $\varepsilon_{0}>|\varepsilon| \beta$, где

$$
\varepsilon_{0}=\min (m, M), \quad m=\left|\inf _{\bar{O}_{1}} a(x, 0)\right|, \quad M=\sup _{\bar{O}_{1}} a(x, 0) .
$$

Итак, утверждение теоремы справедливо при условии $0<|\varepsilon|<\min \left[\left|a_{y}\left(x_{0}, 0\right)\right| /(2 B)\right.$, $\left.\varepsilon_{0} / \beta\right]$.

Очевидно, меняя условия, налагаемые на $a(x, y), b(x, y)$ и их производные в точке $\left(x_{0}, y_{0}\right) \in S \cup Y$, будем получать различные утверждения о локальной ЛЭ-устойчивости или неустойчивости в $\left(x_{0}, y_{0}\right)$ при переходе от уравнения $(1)$ к уравнению $(2)$. Мы ограничились простейшими случаями, рассмотрев три ситуации:

1) $\left(x_{0}, y_{0}\right) \in S,\left(x_{0}, y_{0}\right) \notin S^{*}$

2) $\left(x_{0}, y_{0}\right) \in Y$, т.е. $y_{0}=0$, но $\left(x_{0}, y_{0}\right) \notin S$;

3) $\left(x_{0}, 0\right) \in S \cap Y$.

3. Пример. Покажем на примере, как меняется характер интегральной кривой при пересечении ею сингулярного множества, и применим доказанные теоремы к исследованию пересечения. Рассмотрим нелинейное уравнение $\left(x^{2}+\dot{x}^{2}-2 r\right) \ddot{x}+2 x=0$ или $a(x, y) \dot{y}=1, \dot{x}=1$, где $a=-\left(x^{2}+y^{2}-2 r\right) /(2 x), x \neq 0$, на $S=\left\{(x, y) \in \mathbb{R}^{2}: x^{2}+y^{2}=2 r\right.$, $x \neq 0\}$. По формуле (2)

$$
\frac{d x}{d y}=-\frac{y}{2 x}\left(x^{2}+y^{2}-2 r\right), \quad x \neq 0, \quad S t=S \cup Y,
$$

и по формуле (3a) на $S$

$$
\frac{d^{2} x}{d y^{2}}=-\frac{y^{2}}{x}, \quad x \neq 0
$$

Для применения теоремы 1 рассмотрим $a_{y}=-y / x \neq 0$, если $x y \neq 0$. Следовательно, переход от (1) к (2) ЛЭ-устойчив вне декартовых осей координат. Первьй интеграл имеет вид

$$
I=\exp \left(\frac{y^{2}}{2}\right)\left(\frac{x^{2}}{2}+\frac{y^{2}}{2}-r-1\right)
$$


(нетрудно проверить, что $d I / d t=0)$, т.е.

$$
\mathscr{E}: x^{2}+y^{2}=2(r+1)+C \exp \left(-\frac{y^{2}}{2}\right),
$$

где $C$ - постоянная. Очевидно, уравнение имеет частное решение $x=\sqrt{2(r+1)} \sin (t)$ при $C=0$.

На рис. 1 представлены сингулярное множество и интегральная кривая для $C=0$.

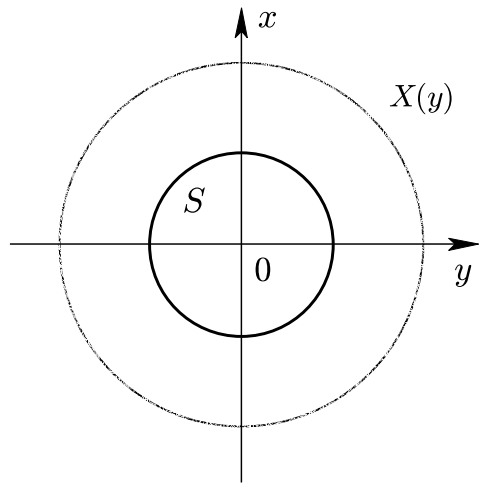

Рис. 1

В этом случае $I(x, y)$ продолжаем на $S$ в классе $C^{1}$.

Для $C=-2$ ситуация представлена на рис. $2 \mathrm{a}$, для $C=-2 \exp (-1 / 8)-$ на рис. $2 \mathrm{~b}, \mathrm{a}$ для $C=-2 \exp (2)-$ на рис. 2 c.
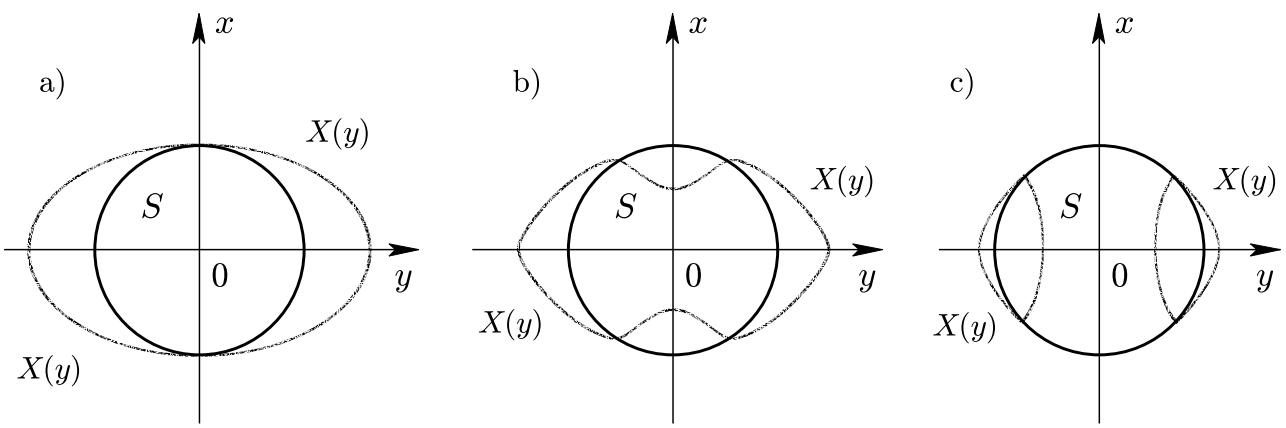

Рис. 2

В этих случаях $\mathscr{E} \cap S \neq \varnothing$. В последнем случае $x(y)$ распадается на две несвязные части. По теореме 1 ЛЭ-неустойчивость может появиться только на пересечении $S \cap Y$. В качестве примера рассмотрим возмущение

$$
\varepsilon b(x, y)=-\varepsilon \frac{y}{2 x}, \quad x \neq 0 .
$$

Получаем уравнение

$$
\left(x^{2}+\varepsilon \dot{x}+\dot{x}^{2}-2 r\right) \ddot{x}=1,
$$


или

$$
\left[a(x, y)-\varepsilon \frac{y}{2 x}\right] \dot{y}, \quad x \neq 0 .
$$

Таким образом,

$$
S^{*}=\left\{(x, y) \in \mathbb{R}^{2}: x^{2}+\varepsilon x+y^{2}=2 r, x \neq 0\right\}
$$

или

$$
S^{*}=\left\{(x, \widetilde{y}) \in \mathbb{R}^{2}: x^{2}+\widetilde{y}^{2}=2 r+\left(\frac{\varepsilon}{2}\right)^{2}, x \neq 0\right\}, \quad y=\widetilde{y}-\frac{\varepsilon}{2} .
$$

Новьй интеграл имеет вид

$$
\exp \left(\frac{y^{2}}{2}\right)\left[x^{2}+y^{2}+\varepsilon y-2(r+1)\right]=\varepsilon \int \exp \left(\frac{y^{2}}{2}\right) d y+C .
$$

$\mathrm{Ha} S^{*} \cup Y$

$$
\frac{d^{2} x^{*}}{d y^{2}}=-y \frac{\varepsilon+2 y}{2 x}, \quad x \neq 0 .
$$

Как нетрудно видеть, теперь

$$
(a+\varepsilon b)_{y}=-\frac{y+\varepsilon / 2}{x}, \quad x \neq 0
$$

Мы рассмотрим только полуплоскость $x>0$, так как в полуплоскости $x<0$ картина симметрична. После элементарных вычислений получаем ситуацию, представленную на рис. 3 .
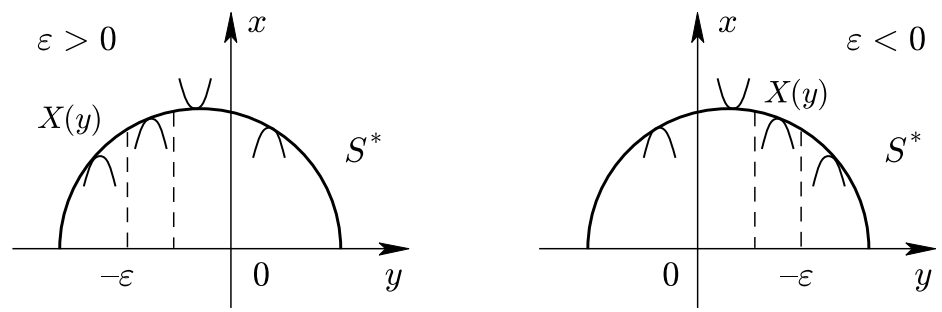

Рис. 3

Таким образом, ЛЭ-неустойчивость появляется только в $\varepsilon$-окрестности точки $\left(x_{0}, 0\right)$ $\in S \cap Y$.

4. Заключение. Локальные экстремумы функций $x(y)$ и $x^{*}(y)$ такие, что функции дифференцируемы в экстремальных точках, могут появляться только на множествах $S \cup Y$ и $S^{*} \cup Y$ соответственно. Поэтому устойчивость экстремумов при переходе от (1) к возмущенному уравнению (2) следует исследовать только на указанных множествах. Теоремы 1-4 обнаруживают некоторые простые достаточные условия устойчивости или неустойчивости.

В заключение авторы приносят благодарность Национальному совету по исследованиям в области функционального анализа и его приложений Республики Италия за финансовую поддержку настоящей работы. 


\section{СПИСОК ЦИТИРОВАННОЙ ЛИТЕРАТУРЫ}

[1] Павлоцкий И. П., Стрианезе М., Тоскано Р. Продолжение решения дифференциального уравнения на сингулярное множество // Дифференц. уравнения. 1998. Т. 34. № 3 . C. $313-319$.

[2] Pavlotsky I.P., Strianese M., Toscano R. Prolongation of the integral curve on the singular set via the first integral // J. Interdisciplinary Math. 1999. V. 2. № 2, 3. P. 101-119.

[3] Pavlotsky I. P., Strianese M. Extension of solution of ODE via the singular set // Nonlinear Anal. 2001. V. 47. P. 4313-4317.

[4] Laserra E., Pavlotsky I.P., Strianese M. Radius of electron as a consequence of Poincaré group // Phys. A. 1995. V. 219. P. 141-158.

[5] Pavlotsky I. P., Strianese M. Some peculiar properties of Darwin's Lagrangian // Int. J. Mod. Phys. B. 1995. V. 9. № 23. P. 3069-3083.

[6] Pavlotsky I.P., Strianese M. Irreversibility in classical mechanics as a consequence of Poincaré groupe // Int. J. Mod. Phys. B. 1996. V. 10. № 21. P. 2675-2685.

[7] Pavlotsky I. P., Strianese M. Minimal distance between the interacting points as a consequence of the singular set of Euler-Lagrange equations // J. Interdisciplinary Math. 2002 (to appear).

[8] Батутин Н.Н., Леонтович Е. А. Методы и приемы качественного исследования динамических систем на плоскости. М.: Наука, 1990.

[9] Павлоцкий И. П., Стрианезе М. Устойчивость сингулярного множества динамической системы // Дифференц. уравнения. 1999. Т. 35. № 3. С. 296-303.

[10] Павлоцкий И. П., Стрианезе М. Устойчивость сингулярного множества обыкновенных дифференциальных уравнений второго порядка относительно $\varepsilon x-$ и $\varepsilon y$-возмущений // Докл. НАН Украины. 2002 (в печати).

[11] Pavlotsky I. P., Strianese M. On the Stability of the Singular Set of the Ordinary Differential Equations of the Second Order with respect to Some Types of Perturbations. Preprint of D.M.I. of University of Salerno (Italia), 2000.

[12] Kaplun Yu. I., Samolenko V. Hr., Pavlotsky I. P., Strianese M. The global theorem on implicit function and its application in the theory of ordinary differential equations // Докл. НАН Украины. 2001. №6. С. 38-41. 